\title{
Laser Based Instrumentation for IAEA Safeguards Verifications
}

\author{
Alain LEBRUN, Victor KRAVTCHENKO, Simone ROCCHI, and Andriy BERLIZOV \\ Department of Safeguards, International Atomic Energy Agency, Vienna International entre, PO Box 100, 1400 Vienna, Austria
}

(Received May 13, 2013)

\begin{abstract}
IAEA verification of the correctness and completeness of State declarations is based on a number of techniques ranging from analysis of open source literature, analysis of commercial satellite imagery, international trade analysis and, of course, field activities conducted by IAEA inspectors and technical staff. A large part of field activities involves instrumentation operated by inspectors or permanently installed at facilities and running in unattended mode. As instrumentation aims at supporting verification of multiple parameters associated with State declarations, multiple techniques are involved, including optical surveillance, sealing and radiation detection, such as neutron coincidence counting or gamma spectrometry. In addition to those techniques considered as traditional for IAEA verification, advanced instrumentation technologies based on lasers have emerged with unique features matching specific safeguards needs, such as the ability to accurately measure distances without physical contact, or extreme sensitivity to trace levels of specific molecules.

The paper describes the various safeguards applications based on lasers and focusses on the difficulties that several technologies faced to meet the unique and stringent requirements of IAEA verification.
\end{abstract}

Key Words: Safeguards verification, Laser range finder, Laser identification, Laser surface authentication, Laser surface mapping

\section{Introduction}

IAEA verification of the correctness and completeness of State declarations is based on a number of techniques ranging from analysis of open source literature, analysis of commercial satellite imagery, international trade analysis and, of course, field activities conducted by IAEA inspectors and technical staff. A large part of field activities involves instrumentation operated by inspectors or permanently installed at facilities and running in unattended mode. As instrumentation aims at supporting verification of multiple parameters associated with State declarations, multiple techniques are involved, including optical surveillance, sealing and radiation detection, such as neutron coincidence counting or gamma spectrometry. In addition to those techniques considered as traditional for IAEA verifications, advanced instrumentation technologies based on lasers have emerged with unique features matching specific safeguards needs, such as the ability to accurately measure distances without physical contact, or extreme sensitivity to trace levels of specific molecules.

The Department of Safeguards of the International Atomic Energy Agency is continuously monitoring the evolution of technologies applicable to field missions carried out by IAEA inspectors in the framework of the implementation of safeguards agreements in States. The applicability of commercially available innovations is systematically evaluated and when necessary development projects are initiated mainly with the voluntary contributions provided by Member States under the system of Member State support programmes to IAEA safeguards. In a limited number of cases, companies or research institutes are contracted by the IAEA to carry out specific development projects.
Development projects have been initiated for eight instruments based on laser technology. They can be segmented in two families of applications depending on whether the laser is used to measure distances or whether spectrometry is involved. The ability to measure accurate distances is used for containment and surveillance applications while unique features of laser spectrometry are focused on detection and characterization of material and substances.

Each of the technologies is described briefly while emphasis is put on the feedback of the experience gained though the development cycle. The purpose of this paper is to share that experience in order to improve future development processes by fully integrating the lessons learned from earlier experiences.

\section{Laser based containment and surveillance techniques}

\subsection{DLR: 3D Laser Range Finder}

The IAEA required an efficient technique to effectively and conclusively record detailed constructional information in support of Design Information Verification (DIV) activities for large and complex industrial nuclear facilities. The IAEA also needed to maintain the continuity of knowledge regarding the verified design of such complex facilities including piping, equipment, cells and buildings by verifying, at the subsequent DIV, that no changes were made. In 2003 the European Commission Joint Research Center (JRC-Ispra, Italy) started the development of the 3D Laser Range Finder instrument composed of a portable commercial 3D laser range scanner (FARO Focus 3D) and a software package (JRC 3DLVS). ${ }^{1)}$ The development has been supported and coordinated by the European Commission support programme. In 2004-2005, the 3DLR 
instrument was successfully evaluated in the field at the Rokkasho Reprocessing Plant (RRP) in Japan and it demonstrated to be a valuable tool to support DIV activities. In 2012 the 3DLR was upgraded to the current version with the introduction of both the FARO Focus 3D scanner and the JRC 3DLVS software package including: data processing, automatic detection of changes, distance measurement tools, visualization and reporting.

The 3DLR is able to build global 3D models with millimetre precision, from scan data taken at different positions in a nuclear facility. It is used as support to DIV activities, providing accurate documentation and maintaining the continuity of knowledge of the verified design, identifying changes in positions and modifications with high accuracy.

Since November 2012 the 3DLR is authorized by the IAEA for use in inspections for DIV activities. Extension of the domain of application is currently being explored as the IAEA is benchmarking applications for containment verification and site surveys of facilities (storages, geological repositories, etc.).

The development of the 3DLR was originated by the commercial availability of compact, accurate, fast and safe 3D laser scanners. Based on the use of a commercial technology for the 3D laser scanner, the application was tuned to the specific safeguards requirements through the development of a dedicated software package for 3D models post-processing and analysis. With 3D laser scanning getting much more affordable a wider range of applications is getting within reach. Involvement of the experts from JRC-Ispra has been instrumental to the success.

\subsection{L2IS: Laser Item Identification System}

IAEA inspectors expressed the need of a system able to assist in identification and tracking $\mathrm{UF}_{6}$ cylinders at the Rokkasho Enrichment Plant (REP), when entering or exiting the process area. The need was also to support verifications of $\mathrm{UF}_{6}$ cylinders against an advance declaration provided by the operator. Since no off-the-shelf solution was available, the alternative option was to engineer a custom system. In 2006 the JRC-Ispra started the development of the Laser Item Identification System (L2IS) instrument. The method is based on the assumption that the detailed shape and micro-relief characteristics of a cylinder are unique and stable enough to serve as the item's identification features. The L2IS is based on the laser triangulation technique offering a highly modular solution, enabling the integration of a sensor optimized towards various requirements (working range and depth resolution). The development has been supported and coordinated by the European Commission support programme. The L2IS is based on the combination of both attended and unattended monitoring methods to identify $\mathrm{UF}_{6}$ cylinders entering and exiting a processing area. As a complement to traditional video surveillance, the L2IS provides a fast, accurate and reliable method to perform $\mathrm{UF}_{6}$ cylinder inventory in process and storage areas. The L2IS is based on two types of laser scanner units:

- L2IS Unit 1 (attended unit): used by inspector to populate the database upon delivery of the cylinders at the facility (yearly base);

- L2IS Unit 2 (unattended unit): installed in a strategic point of the process, thus monitoring all entering and exiting cylinders.

The L2IS has been designed and built specifically for REP to assist inspectors in identifying and tracking $\mathrm{UF}_{6}$ cylinders of the tree types of cylinder in use at the facility: $30 \mathrm{~B}, 48 \mathrm{Y}$ and IPC. ${ }^{2)}$ Since November 2012, the L2IS has been autho- rized by the IAEA for safeguards use at REP. Together with Non Destructive Assay features and surveillance cameras, the L2IS is envisaged as a component of a safeguards system that could be built around operator's accountancy scales at other enrichment plants.

Once engineered and developed by JRC-Ispra, the L2IS system has been successfully installed in REP on trial and evaluated, since 2007, on a large set of verification and reference scans acquired from three types of $\mathrm{UF}_{6}$ cylinders.

Although there were no false positive or false negative cases, field tests have shown cases when the cylinders were detected, but could not be identified. Therefore, in these cases, the L2IS should be complemented by inspector review of the data and with other complementary safeguards procedures. With further testing, and minor changes on the setup if needed, the L2IS system can be used for other types of cylinders and/or in other enrichment facilities.

\subsection{LSAS: Laser Surface Authentication System}

In its current practice, the IAEA widely uses simple metal cap seals, which are additionally modified by applying individual unique engraved patterns on their internal surface to serve as a reference for subsequent identification. The creation of such signatures on IAEA metal seal caps, their referencing and verification at the IAEA laboratories are still based on manual and labour-intensive procedures. The IAEA has been looking for a technology enabling replacement of manually created signatures and utilization of automated recording of references and verification data of metal seals. One of the options was to use intrinsic features of the seal caps surfaces microstructure as a unique signature. LSAS, a new emerging technology that had already demonstrated reliable results in the unique identification of different types of surfaces was selected for a feasibility study. ${ }^{3)}$ The technology was originally developed in the early 2000s, at Imperial College London, UK and, later, commercialized by the UK based company Ingenia Technology Ltd.

Numerous experiments and test conducted by Ingenia Technology Ltd on metal seals demonstrated the ability of the technology to uniquely identify brass and copper surfaces of metal seal caps, ${ }^{4}$ which led to the development and delivery of two robotic systems currently installed at the IAEA Seals Laboratory. Those systems are designed for fully automated referencing and verification of metal seals. Unfortunately, a third party vulnerability assessment (VA) concluded that the LSAS delivers unacceptably high false positives and false negatives verification results. In particular, unacceptable sensitivity was demonstrated for slightly corroded or damaged seals, which is often the case when seals are returned after several months at nuclear facilities. The overall VA conclusion was not to recommend LSAS technology as an application for metal surfaces verification. As a consequence, LSAS is not currently used for metal seals verification and no alternative technology has been identified to automate the processing of IAEA metal seals.

The main lesson learned from this development is that an early third party vulnerability review (VR), carried out at the very first prototype phase would probably have detected the vulnerability of the technique and saved those resources expended on the development and production of the LSAS robotic instruments. Such early vulnerability review has now been integrated into the procedures applied for development and authorization of equipment involved in IAEA safeguards verification. 
2.4 LMCV: Laser Surface Mapping System for Containment Verification

The IAEA has identified a requirement for techniques to verify integrity of containers of nuclear materials. Such technology would be used as an alternative or as a complement to the conventional sealing technologies. A Laser Surface Mapping (LSM) technology ${ }^{5)}$ has been identified for a feasibility study. The LSM accurate mapping of a surface results in digital images that can easily be stored and kept for reference. Tampering attempts will result in substantial differences between the references and subsequent rescans.

The JRC-Ispra has been working on this task in the framework of the European Commission support programme to IAEA safeguards. A feasibility study explored two directions for scanning of the entire surface of a container ${ }^{6}$ and for verification of the integrity of welds bonding together container's parts. ${ }^{7}$ Two different instrument prototypes have been developed. The LMCV-C focused on the verification of the integrity of the entire surface of the container. The LMCV-W was devoted to the verification of the weld integrity. Internal evaluation of both instruments showed promising results. However, only the LMCV-W was finally recommended by the third party vulnerability assessment for IAEA Safeguards application. After confirmation of the operational need for a technology to verify specific casks used to store irradiated nuclear fuel, The JRC and the IAEA worked on the final design of an instrument for verification of these cask welds. The instrument was completed after extensive field tests in 2012. Currently, the LMCV-W instrument is authorized for inspection use and routine implementation is planned for end of 2013.

Implementation of LMCV-W for verification of spent fuel casks will result in significant efficiency gains as lower workload for sealing replacement and verification will be required. Thanks to careful design of the data security features of the instrument, additional efficiency benefits are expected from the possibility to involve facility operators, in the framework of the enhanced cooperation.

There are two lessons learned during that development. Even for well-established and commercially available technologies, when those are intended for Safeguards applications, third party vulnerability assessment are crucial to ensure credibility. Extensive field trials are necessary for finalizing and validating robust instrument designs.

\section{Laser based detection and analysis techniques}

\subsection{HFLS: Hydro Fluorine detector Laser System}

The IAEA identified a strong need to establish technical capability to detect undeclared handling of $\mathrm{UF}_{6}$. The HFLS development was initiated to respond to that need as Hydro Fluorine (HF) had been identified as a strong indicator of the presence and manipulation of $\mathrm{UF}_{6}$. From 2006, the General Physics Institute (GPI) in Moscow cooperated with Canberra Industries to develop an absorption spectrometer based on Tunable Diode Lasers to detect and measure parts per billion levels of HF in air. ${ }^{8}$ The prototype instrument developed by GPI and Canberra proved to meet sensitivity requirements but faced stability problems which would have resulted in unacceptable false positive alarms. In the meantime, a commercially available instrument had been proposed by Picarro Inc.. The claimed characteristics of the instrument were assessed positively despite lower sensitivity compared to the GPI instrument. Before engaging resources in the procurement of a Picarro instrument for testing, a re-evaluation of the need was conducted in 2010. It demonstrated that, although HF is in- deed an indicator of $\mathrm{UF}_{6}$, its presence is unfortunately also confirmed at ppb levels in conjunction with numerous other industrial processes. Therefore, the final conclusion was that $\mathrm{HF}$ is not a workable indicator. A decision was made not to pursue HF detection for detection of undeclared activities.

The HFLS development project brought several lessons. That particular development had been driven by the technology availability more than through thorough analysis of the need. Development through an academic institute was concomitantly initiated with ongoing development in a specialized company that had not been identified. It demonstrates that sufficient efforts must be allocated both robust need assessments and to complete market surveys through continuous technology foresight. Such elements have since been incorporated in procedures applied for development and authorization of equipment involved in IAEA safeguards verification.

3.2 UFLS: Uranium hexa-Fluoride detector Laser System The IAEA identified a need to establish a technical capability to perform accurate isotope ratio measurements on $\mathrm{UF}_{6}$ samples at enrichment sites. The UFLS development was initiated to cover that need as laser absorption spectrometry supported by tunable lasers had been identified as a potential candidate to measure uranium enrichment with high accuracy on $\mathrm{UF}_{6}$ samples. The General Physics and Kurchatov institutes in Moscow published a paper in $2006^{9)}$ which established the experimental foundations of the feasibility of an accurate uranium enrichment by means of laser absorption spectrometry. Subsequent work under IAEA contracts lead to the development of an experimental setup. Experimental proof of principle for the measurement at requested accuracy was brought in 2007 and was reported in reference. ${ }^{10)}$ Work continued to optimize the experimental set up by integration of better performing components and mastering memory effects. ${ }^{11)}$ In the meantime, the German support programme to IAEA safeguards supported the UFLS development in contracting the company Laser Components $\mathrm{GmbH}$. to develop and manufacture specific Lead-Salt Lasers at $7.7 \mu \mathrm{m}$. Those lasers still required liquid nitrogen cooling but achieved unprecedented tuning ability. Unfortunately those performances suffered lack of stability in time.

External factors impacted the project dramatically. Quantum Cascade Lasers (QCL) emerged as a credible alternative to diode lasers thus making the diode laser development obsolete. Further, a patent granted in 2009 induced limitation in the ability of the IAEA to engage directly in the development. Experimental proof of principle had been achieved in 2007 in a laboratory environment. Extrapolation to a deployable instrument appeared more challenging than anticipated due to the extremely high performances of the spectrometric measurement required to match the requested accuracy.

Combination of those factors led to the discontinuation of efforts to further develop the method.

The operational need remains for onsite accurate determination of uranium enrichment on $\mathrm{UF}_{6}$ samples. Only a low level of technology survey continues to be carried out on laser spectrometry. The IAEA is also carefully investigating alternative options including on site mass spectrometry.

This development project was a great scientific adventure. Its risky nature was known from the beginning as the measurement itself was identified as very challenging. The project suffered from lack of anticipation of the technological evolution of lasers. Early identification of the rapid progression of quantum cascade lasers could have saved significant resources spent to improve the lead-salt tunable diode laser technology 
which was next to obsolescence. IAEA resources for the project were at the same time insufficient and excessive. Insufficient, because assigned resources were covering only the establishment of the proof of principle under the assumption that another source of funding would take over the system integration. Even though rather limited, the IAEA investment was excessive because, in addition to accepted risks induced by the scientific challenges, organizational difficulties evidenced by the fact that the technology had been patented were representing a high risk for that investment. This case is the materialization of the necessity to perform careful risk assessments before engaging into even the most appealing scientific development.

\subsection{HHRM: HandHeld Raman Instrument}

Realizing that the rapid and reliable in-field detection and identification of special chemical compounds and materials can significantly contribute to early detection of undeclared nuclear activities, the IAEA has started evaluation of several laser based chemical identification technologies, among which are the Raman spectroscopy and laser induced break-down spectroscopy (LIBS). The Handheld Raman Instrument (HHRM) under evaluation is a commercially available First Defender RM handheld spectrometer manufactured by Thermo Scientific. This selection was made based on the market search using such criteria as form factor, weight, method of use, certification of ruggedness, scope of material library, power options and operating environment conditions. The First Defender RM has suitable portability, ruggedness, environmental protection and weight characteristics. It provides the analysis capability in the point-and-shoot and vial geometries. The identification of chemical compounds and mixtures is performed using a robust chemometrics analysis procedure, which is based on an on-board library pre-loaded with $\sim 7000$ Raman responses.

Although being extensive, the library contains a limited number of compounds of safeguards relevance. It is therefore very essential that the instrument's firmware provides an easyto-use library upgrade capability, allowing adaptation of the HHRM to the safeguards specific needs. For instance, within a recent study conducted jointly with the EC JRC Institute for Transuranium Elements (Karlsruhe, Germany), a representative collection of more than 40 uranium ore concentrate (UOC) samples, covering a wide range of industrial "yellow cakes" of different types, origin and purity, was used to populate the instrument's library. A subset of UOC samples was then measured as "unknowns" to test the identification capability of the upgraded instrument. The accuracy of the library matches was shown to be very good for uranyl peroxides and sodium diuranates, for which the identification results could be delivered in a few seconds. UOC samples, containing ammonium diuranates or uranyl hydroxides as a major compound, could be identified in a few minutes with a decreased identification probability compared to the uranyl peroxide and sodium diuranate based "yellow cake". The uranium dioxide and tri-uranium octa-oxide samples could not be analyzed with the instrument. A separate study showed a potential of the instrument for a quick and reasonably accurate quantitative determination of the uranium concentration in UNH solutions with known acidity. The HHRM is moving towards the final authorization for safeguards use and is going to be a useful tool in complementary access missions aimed at detection of signatures of undeclared nuclear material and activities.

\subsection{PHHL: Portable Hand-Held LIBS}

The Portable Hand-Held LIBS Instrument (PHHL) has been under development by the National Research Council Canada since 2006. The development has been supported and coordinated by the Canadian Support Programme to IAEA safeguards. Since December 2009, when the first prototype PHHL was delivered to the IAEA, the instrument has undergone several upgrades primarily aimed at the increased performance of both hardware and software. A workable version of the PHHL was delivered to the IAEA in 2011. The instrument was built up from commercially available parts, such as Nd:YAG Kigre Inc. laser (10 $\mathrm{mJ}$ per pulse, max repetition rate $20 \mathrm{~Hz}$ ) and Avantes AvaSpec 2048 fiber optic spectrometer, and equipped with a dedicated custom made acquisition and powerful chemometrics analysis software. The "training" feature of the software allows adaptation of the instrument to different types of materials, which was envisaged to be done for a variety of safeguards relevant chemical compounds and materials. Though, due to the instability of the laser unit that repeatedly showed a significant power drop over a short period of time, only little progress has been made in this direction since 2011. The intended application area of the PHHL is overlapping with the Raman HHRM spectrometer and complements it with regard to material types, with more emphasis on the elemental composition and impurity content. The added value of the method for safeguards still has to be evaluated, further explored and proved in comparison with well-established analytical techniques such as X/Ray Fluorescence and Raman spectroscopy. A major effort is still need to be applied in order to bring this technology to the level of real practical application. One of the major lessons learnt, therefore, is that the evaluation of maturity and readiness of a new technology prior to its practical implementation is very essential. It is especially important in view of the very limited resources that the Agency can devote to the research and development type of activities.

\section{Conclusion}

In the last decade, the IAEA has undertaken a total of eight specific projects meant to incorporate laser based advanced instruments in the arsenal of instruments for field use by IAEA nuclear inspectors. Only half of them (3DLR, L2IS, LSCV, HHRM) succeeded fully and are currently deployed for actual implementation of IAEA safeguards. At a late stage in the development process the Laser Surface Authentication System (LSAS) proved to be too vulnerable to be considered a credible verification instrument. The HF Laser System (HFLS) development was not fully successful and although the HF detection became commercially available, the technology was finally not implemented. Technological difficulties associated with the miniaturization of the YAG ablation laser have not been yet overcome in the case of the PHHL (handheld LIBS) and the UF $_{6}$ Laser System (UFLS) could not reach readiness for field deployment although the scientific proof of principle has been established.

As again illustrated by the examples above, development of instrumentation is confirmed to be a lengthy and risky business. Many lessons have been learned and integrated into IAEA processes for development of the instrumentation supporting the IAEA mandate for verification relevant to the Treaty of Non Proliferation. A more structured decision making and project managing procedure has been established in 2011. It requires comprehensive evaluations of risks, vulnerabilities and alternative options. It incorporates related formal 
decision steps. The IAEA is also conducting systematic technology foresight the main objective of which is to inform on the emergence of technologies suitable for IAEA safeguards. Its second objective is to inform the decision on initiating or not specific developments by providing early identification of commercially available technologies. Indeed our experience is that, although less attractive from a scientific stand point, adapting commercially available technologies to IAEA safeguards applications does have higher chances of success compared to project that include technology enabling which are much more risky.

\section{References}

1) V. Sequeira, G. Bostroem, M. Fiocco, J. Goncalvez, S. Poirier, and B. Wishard: Proc. ESARDA, Aix-en-Provence, 2007 (OPOCE, 2007) p.1.

2) A. Busboom, V. Sequeira, D. Langlands, B. Wishard, and S. Poirier: Proc. ESARDA, Aix-en-Provence, 2007 (OPOCE, 2007) p.1.

3) J. D. R. Buchanan, R. P. Cowburn, A. Jausovec, D. Petit, P. Seem,
G. Xiong, D. Atkinson, K. Fenton, D. A. Allwood, and M. T. Bryan: Nature 436 (2005) 475.

4) M. McGlade S. Poirier, H. Undem, and M. Zendel: IAEA Proceedings Series (2007) IAEA-CN-148/123.

5) E. Bovisio, V. Sequeira, M. Heppleston, and J. G. M. Goncalves: Proc. ESARDA (2003) 108.

6) A. Bosboom, G. Bostroem, and V. Sequeira: Proc. ESARDA, Aixen-Provence, 2007 (OPOCE, 2007) p.1.

7) A. Busboom and V. Sequeira: Proc. SPPRA, Innsbruck, 2007 (ACTA Press, Anaheim, 2007) p.7.

8) G. Grigoriev, A. Lebrun, A. Mantz, L. Mechold, A. Nadezhdinskii, N. Peter-Stein, V. Ryzhikov, F. Tittel, and M. Zendel: Proc. TDLS (2009) 21.

9) A. G. Berezin, S. L. Malyugin, A. I. Nadezhdinskii, D. Yu. Namestnikov, Ya. Ya. Ponurovskii, D. B. Stavrovskii, Yu. P. Shapovalov, I. E. Vyazov, V, Ya. Zaslavskii, Yu. G. Selivanov, et al.: Spectrochim. Acta A Mol. Biomol. Spectrosc. 66 (2007) 796.

10) A. Lebrun, V. Ryzhikov, M. Zendel, A. Nadezhdinskii, G. Grigorev, and L. Mechold: Proc. INMM annual meeting (2008) 1782.

11) A. I. Nadezhdinskii, N. Y. Ponurovskii, and S. P. Kadner: US Patent 7535006 (2009). 